This report was prepared as an account of work sponsored by the United States Government. Neither the United States nor the United States Atomic Energy Commission, nor any of their employees, nor any of their contrac. cors, subcontractors, or their emplevees, makes any warranty, express or im. plied, or assumes any legal liability or responsibility for the accuracy. com. pleteness or usefulness of any information, apparatus, product or process dis. closed, or represents that its use would not inlringe privately owned sights.

Printed in the United States of Ametics. Availabte from National Technical Intommaren Service

U. S. Depurtment of Commerce 5285 Port Roval fiow Soringlield. Virginia 22451

Priet: Printed Copy \$4.00: Microliche $\$ 0.95$ 


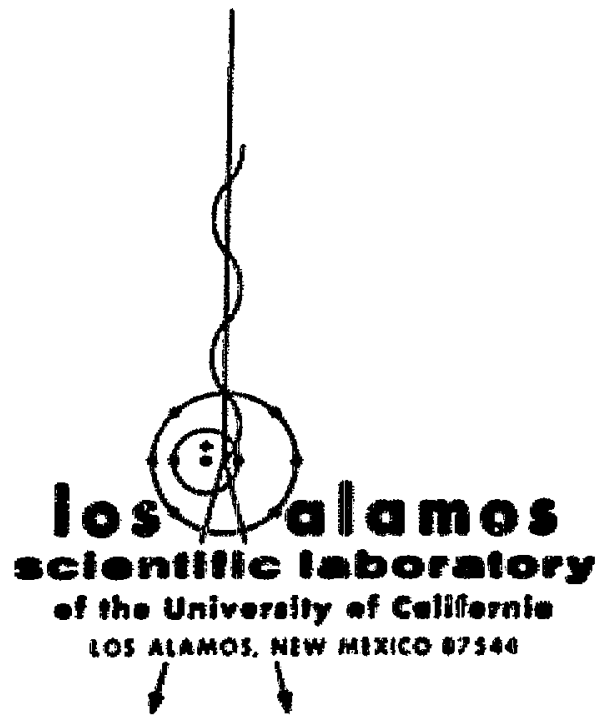

\title{
An Iterative Procedure for Alignment in Underground Nuclear Testing
}

by

\author{
E. A. Kam
}

morlet

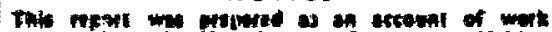

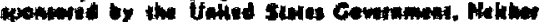

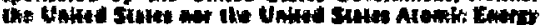

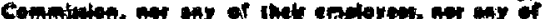

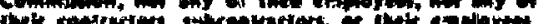

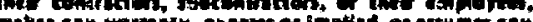
me

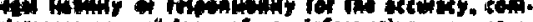

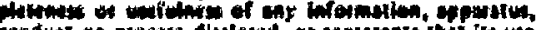

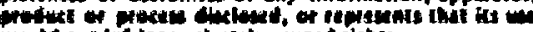

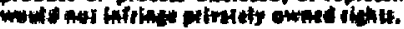



$\mathbf{d}_{\mathbf{l}}=$ outer beam diameter
$d_{2}=$ inner beam diamerer
む = vector from origin of $x y z$ to origin of $x^{\prime} y^{2} z^{\prime}$
$8=$ magnitude of $\overrightarrow{\mathbf{G}}$
h $=$ frustum height
1 payoîf function to be minimized
$K=$ penaity constant
L. = iength of support leg edge
$\overrightarrow{0} \quad=$ vector from origin of XYZ to arigin of $x^{2} y^{\prime} z^{\prime}$

$\vec{p}=$ vector from arigin of XYZ to end point of support leg edfe

$\overrightarrow{\mathbf{a}} \quad=$ vector defining location of support leg edze relative to origin of $x^{\prime} y^{\prime} z^{\prime \prime}$

$\vec{R} \quad$ - vector from origin of XYZ to a point on the frustum surface

$r_{c} \quad=$ perpendiculat distance from the pipe centertiue to an arbikary point on the circumicrence of the small end of the frustum

$r_{\text {ef }}=$ perpendicular distance from the pipe centeritine to an arbitrary point on the inustum scifface

$\boldsymbol{r}_{\text {cfmin }}=$ minimum value of $\boldsymbol{r}_{\mathrm{cf}}$

$t_{\mathrm{cmax}}=$ maximum value of $r_{\mathrm{c}}$

rf $=$ radius of an arbitrary frustum cireular cross section

$$
\begin{aligned}
& r_{0} \quad=\text { outer beam radius } \\
& r_{1}=\text { radius of the large end of the frustum } \\
& x_{2}=\text { radius of the small end of the frustum } \\
& \text { s } \quad=\text { unit step function } \\
& \overrightarrow{\mathbf{S}} \quad=\text { vector directed along the support leg edge } \\
& T=\text { orthogonal transformation matrix from } \\
& \text { xyz to XYZ system } \\
& \vec{U} \text { = vector from origin of XYZ to origin of } \\
& \text { xyz } \\
& \vec{v} \quad=\text { vector from origin of } x y z \text { to a point on } \\
& \text { the frustum surface } \\
& \text { w = width of a suppor leg } \\
& X Y Z=\text { coordinate system with origin at the pipe } \\
& \text { center } \\
& x y z=\text { coordinate system fixed to the fnustum }
\end{aligned}
$$

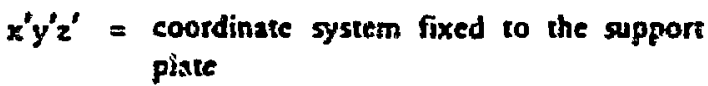

$$
\begin{aligned}
& z_{p} \quad=\text { perpendieular distance from the large end } \\
& \text { of the frustum to an arbitrany cross- } \\
& \text { sectional plane } \\
& \beta, \gamma_{3} \theta=\text { Euler rotation angles } \\
& \downarrow \quad=\text { angle measured in a cross-sectional plane } \\
& \text { of the frusum } \\
& \begin{array}{l}
{[]=\text { column vector }} \\
()_{x}=x \text {-coordinate of a vector }
\end{array} \\
& {[\mathrm{XYYZ}=\text { vector in } X Y Z \text { coordinates }}
\end{aligned}
$$




\title{
AN ITERATIVE PROCEDURE FOR ALIGNMENT \\ IN UNDERGROUND NUCLEAR TESTING
}

by

E. A. Kem

\begin{abstract}
A new method is presented for determining the mounting geometry for a frustum within the urnel of an undergound nuclesr testing site. This method is based on a sequence of linear iterations in conjunction with a Davidon iterator for finding the minimum of a function of several parameters. Successful convergence of the method has been demonstrated on a time-sharing CDC 6600 computer. We believe this method could be generalized to other similar alignment problems.
\end{abstract}

\section{INTRODUCTION}

In the past, difficult geometric alignments associated with experiment packsages in underground nuelear tests have been accomplished at the Los Alamos Scientific Laboratory by tedious drafting layouts. Recently, it was decided to atrempt a computer solution of an extremely difficult alignment problem involving the placement of the frustum of a cone in the test tunnel. Early in the analysis it was concluded that an iterative-type approach would offer the best solution for this problem. Using a series of linear iterators in conjunction with a Devidon iterasos," we successfully solved the problem with a CDC 6600 time-sharing computer system. This report describes the prob!em and presents the method of sokution. To avoid security classification of this report, specific dimensions ant computer results are not included.

\section{DESCRIPTION OF THE PROBLEM GEOMETRY}

A frustum of a cone is mounted in the test pipe as shown in Fig. 1. The beam diameters $d_{1}$ and $d_{2}$ ivepend

\footnotetext{
"W. C. Ouvidon, "Varisble Metric Method for Minimisution," AEC Resereh and Development repaze ANL-5990 (1959).
}

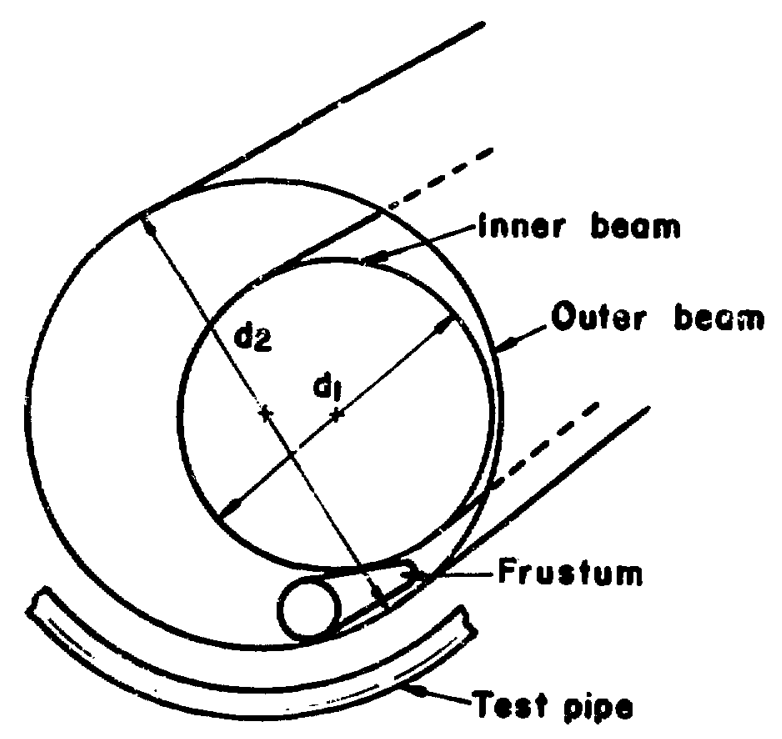

Fig. 1.

Location of frustum within a sadiation pattern. 
on the distance from the radiation source. The frustum is to remain in contact with the large or outer beam and is to be tangent at some point to the small or inner beam. This inner beam tangency requirement is imposed so that the frustum does not interfere with experiments farther down the test pipe. In addition, the centerline of the frustum is to be inclined at some given angle $\phi$ with the test pipe centerline. The frustum is mounted on a flat plate that, in turn ${ }_{\mathrm{r}}$ is supported on the wall of the test pipe by four support legs perpendicular to the mounting plate. The orientation and location of the frustum relative to the mounting plate and the location of the support legs relative to the mounting $F$ 'ate are fixed quantities (see Fig. 2). The basic problem is to determine the mounting locations and the dimensions of the four support legs on the test pipe so that the frustum satisfies the required alignment geometry.

\section{ITERATIVE SOLUTION OF THE FRUSTUM ALIGNMENT PROBLEM}

The first step in the solution is to determine how the frustum should be located and aligned relative to the inner and outer beans. For this purpose we define an XYZ-coordinate system with origin at the pipe center, with the Z-axis directed along the pipe centerline and the $X$-axis directed vertically upward (see Fig. 3). We also define an xyz-coordinate systein fixed to the frustum with the $x$ - and $y$-axes in the plane of the frustum bast (large end of frustum) and the origin at the point where

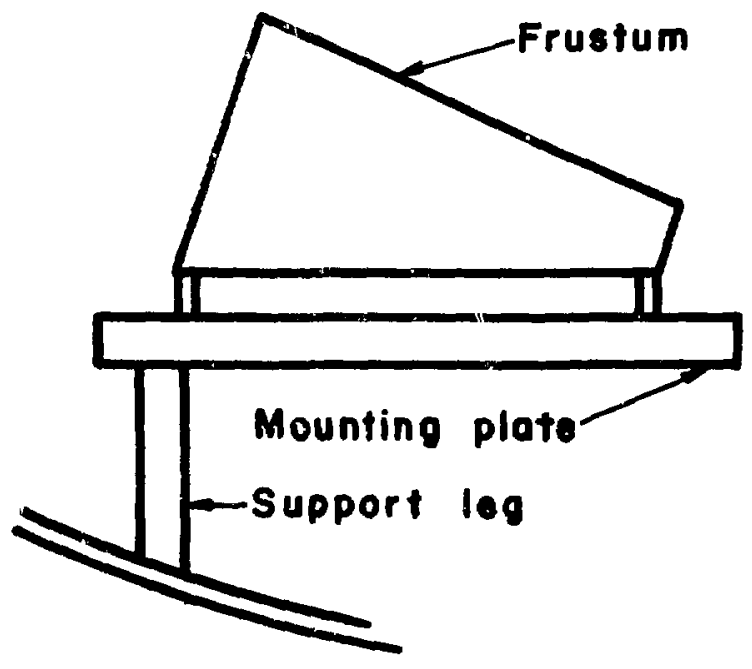

Fig. 2. Frustum mounting geometry. the outer beam and the frustum base are tangent (see Fig. 3). The origins of the $X Y Z$ and $x y z$ systems lie in the same cross-sectional plane of the pipe. The frustum is shown in Fig. 3 at the bottom of the pipe with the $x-, y-$, and $z$-axes respectively parallel to the $X-, Y$-, and Z-axes to :implify the geometry of the figure.

When the frustum is located and oriented correctly, the: origin of the xyz system will not generally lie at the bottom of the pipe nor will the $x-, y-$, and $z$-axes be parallel to the $X-, Y$-, and $Z$-axes, respectively.

Because the xyz-coordinate system is fixed to the frustum, the orientation and position of the frustum will be determined by the origin and orientation of the xyz-coordinate system relative to the $X Y Z$ system. If the geometry of Fig. 3 is used as a starting point, the orientation and position of the xyz-coordinate system can be defined by a translation and three Euler angle rotations in the following manner.

(1) Translate the origin of the xyz sysiem through the angle $\theta$ along the large beam circumference and simultaneously lotate the coordinate system about the z-axis so that the $x$-axis still points toward the center of the pipe (Fig. 4). In effect, the xyz system has been translated and /rotated through an angle $\theta$ in the negative sense (righthand rule) about the 2 -axis.

(2) Rotate the new xyz system in a negative sense about the $x$-axis through the angle $\beta$.

(3) Rotate the new xyz system in a positive sense about the $y$-axis through the angle $\gamma$.

This rranslation through the angle $\theta$ and the Euler rotation angles $\theta, \beta$, and $\gamma$ defines the position arid orientation of the xyz system (hence the frustum) relative to

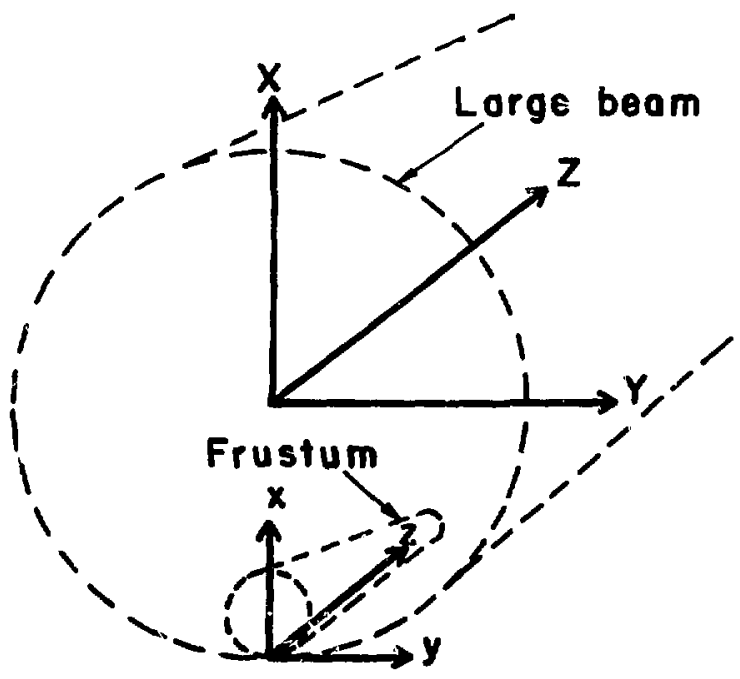

Fig. 3.

Definition of the $X Y Z$-and $x y z-c o o r d i n a t e ~ s y s t e m s$. 


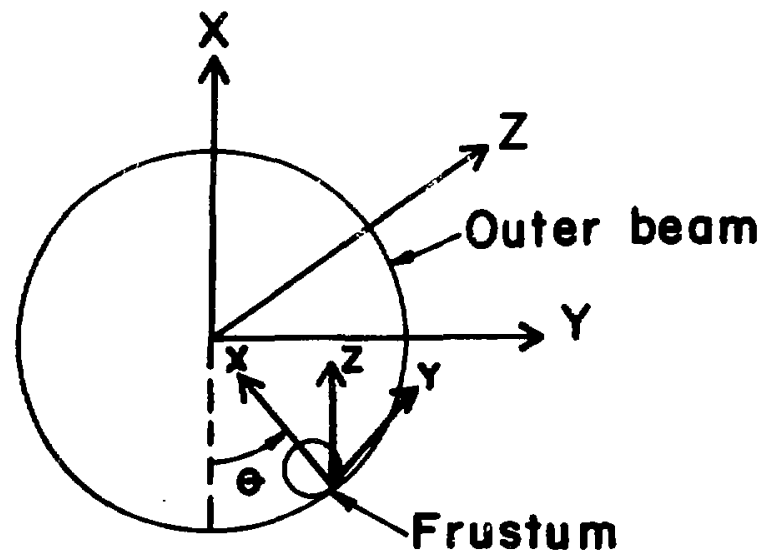

Fig. 4.

Displacement and rotation of the $x y z$ system tbrough the angle $\theta$.

the $\mathrm{XYZ}$ system. Thus, the entire alignme it procedure centers around the determination of the angles $\theta, \beta$, and $\gamma$ so that the frustum satisfies the specified requirements.

(1) The frustum base and the end of the frustum opposite the base are tangent to the large beam.

(2) The centerline of the frustum is inclined to the pipe centerline at some angle $\phi$.

(3) The surface of the frustum is tangent at some point to the inner beam.

A direct method for finding the angles $\theta, \beta$, and $\gamma$ to satisfy the above three requirements did not immediately present itself; therefore, the following iterative approach wos used.

(1) Estimate the pipe station at which the smaller end of the frustum (end opposite the base) is tangent to the outer beam and the pipe station at which the surface of the frustum is tangent to the inner beam. This establishes an estimate for the beam diameters at the corresponding tangency points.

(2) Guess the angles $\theta, \beta$, and $\gamma$.

(3) Perform a linear iteration of the angle $\gamma$ so that the end of the frustum oppositc the frustum base is tangent to the outer beam.

(4) Perform a lincar iteration on the angle $\beta$ so that the centerline of the frustum is inclined by the desired angle $\phi$ to the pipe centerline. For each new angle $\beta$, recum to step 3 and reiterate on the angle $\gamma$.

(5) Perform a linear iteration on the angle $\theta$ so that the surface of the frustum is tangent to the inner beam at sorne foint. For each new angle $\theta$, return to steps 3 and 4 to reiterate on the angles $\gamma$ and $\beta$.

(6) Compute the actual pipe stations where the smaller end of the frustum is tangent to the outer beam and the surface of the frustum is tungent to the inner beam. If the actual pipe stations differ by more than 0.0001 in. from the estimated pipe stations, set the estimated pipe stations equal to the actual pipe stations and return to step 2 of the iteration.

The above iteration procedure has proved successful in determining the angles $\theta, \beta$, and $\gamma$ and thus establishes the location and orientation of the frustum. The mathematical details associated with each of the above steps were purposely omitted so as not to obscure the basic iterative procedure.

The beam diameters along the pipe for both the outer and the inner beams increase as the distance down the pipe increases. Therefore, we must know the exact pipe stations at which the tangency points described in step 1 occur so that the outer and inner beam diameters can be established at these points. Because the pipe stations for the tangency points are not known initially, they must be estimated and then iterated. Note in step 6 that the iteration loop on the pipe stations is closed by setting the estimated pipe stations equal to the corresponding actual pipe stations at which the tangency points are computed to occur.

Very simple linear iterations are used in steps 3,4 , and 5 to determine the angles $\gamma, \beta$, and $\theta$. Consider a linear iteration on the angle $\beta$ where it is desired to obtain a specific inclination angle $\phi$ as an example. Let

$$
\begin{aligned}
& d=\phi \text { desired }-\phi \text { actual, } \\
& d_{n}=\text { value of } d \text { on the isth iteration, }
\end{aligned}
$$

and

$$
\beta_{n}=\text { value of } \beta \text { on the nth iteration. }
$$

To compute $\beta$ for the $(n+1)^{\text {th }}$ iteration, compute

$$
\text { slope }=\frac{\theta_{n}-\theta_{n}-1}{d_{n}-\frac{d_{n}-1}{}} \text {. }
$$

Assume that this slope remains constant over the $(n+1)^{\text {th }}$ iteration, i.e.s

$$
\text { slope }-\frac{B_{n+1}-B_{n}}{d_{n+1}-C_{n}} \text {. }
$$

It is desired that $d_{n+1}$ be zero on the $(n+1)^{\text {th }}$ iteration. Serting $d_{n+1}=0$ in Eq. (2) and solving for $\beta_{n+1}$ gives

$$
t_{n+1}=n_{n}-\operatorname{seps}\left(d_{n}\right) .
$$


Iteration for $\beta$ continues until the absolute value of $d$ becomes less than a prespecified tolerance. The linear iterations on ' $\gamma$ and $\theta$ are performed in the same manner.

In step 3 of the iteration, we must determine the angle $\gamma$ so that the small end of the frustum (end opposite the base) is tangent to the outer beam circumference. This is accomplished by defining a vector $\overrightarrow{\mathbf{R}}$, which locates a point on the circumference of the small end of the frustum relative to the center of the pipe (see Fig. 5). Let the $X-, Y$, , and $Z$-components of $\vec{R}$ be denoted by $R_{X}, R_{Y}$, and $R_{Z}$, respectively, and let $r_{c}$ be the distance of a given point on the small end of the frustum from the pipe center. Then it follows that

$$
\mathbf{r}_{c}=\left(R_{X}^{2}+R_{Y}^{2}\right)^{1 / 2}
$$

The value of $r_{c}$ will vary as $\vec{R}$ moves around the small end of the frustum. The maximum value of $r_{c}, r_{c m a x}$, defines the point on the smaili end of the frustum that is farthest from the center of the pipe. If $\mathrm{r}_{\mathrm{cmax}}$ is smaller than the outer beam radius, the small end of the frustum lies inside the outer beam, whereas if $\mathbf{r}_{\mathrm{cmax}}$ is langer than the outer beam radius, a portion of the small end of the frustum lies outside the outer beam. For the small end of the frustum to be tangent to the outer beam, the $r_{\text {cmax }}$ must be equal to the outer beam radius. Linear iteration on $\gamma$ is therefore performed until $r_{\mathrm{cmax}}$ is within $10^{-8} \mathrm{in}$. of the outer beam radius.

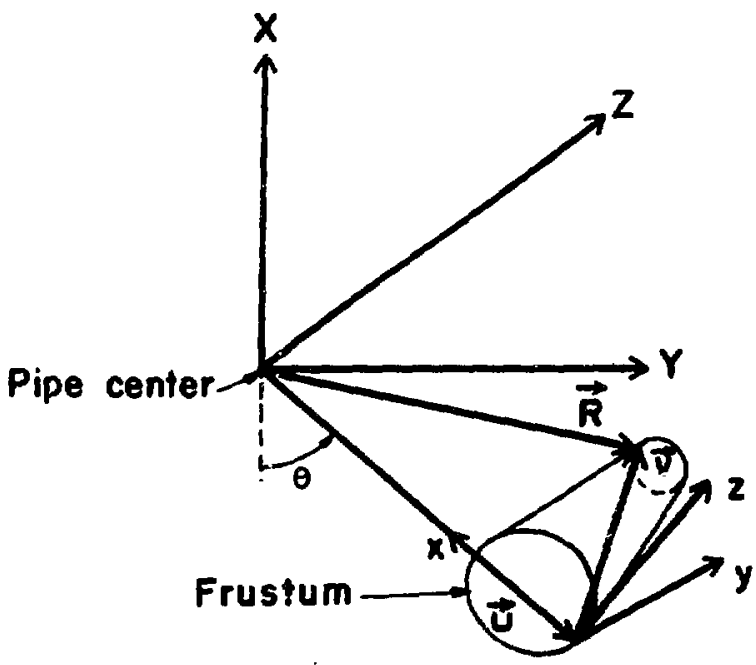

Fig. 5.

Vector $\vec{R}$ from pipe center to point on circumfertnce of small end of frustum.
We compute $\mathbf{r}_{\mathrm{cmax}}$ for a given angle $\boldsymbol{\gamma}$ as follows. From Fig. 5 we have

$$
\overrightarrow{\mathbf{R}}=\overrightarrow{\mathrm{U}}+\overrightarrow{\mathrm{V}}
$$

where

$$
\begin{aligned}
& \vec{U}= \text { the vector from the origin of the } X Y Z \text { system to } \\
& \text { the origin of the } x y z \text { system }
\end{aligned}
$$

and

$\vec{v}=$ the vector from the origin of the xyz system to a point on the small end of the frustum.

The vector $\vec{U}$ is easily established because the origin of the $x y z$ system lies in the $X Y$ plane. Referring to Fig. 5 and letting $r_{0}$ be the radius of the outer beam, we note that

$$
U_{X}=-x_{c}, \quad \cos \theta
$$

and

$$
u_{\gamma}=r_{0} \sin \theta .
$$

The vector $\vec{\nabla}$ is most easily determined in the xyz system and is then transformed into the $X Y Z$ system. Here we consider Fig. 6 where the vector $\vec{V}$ is shown equivalent to the sumi of the three vectors, $\vec{V}_{1}, \vec{V}_{2}$, and $\vec{V}_{3}$, and the

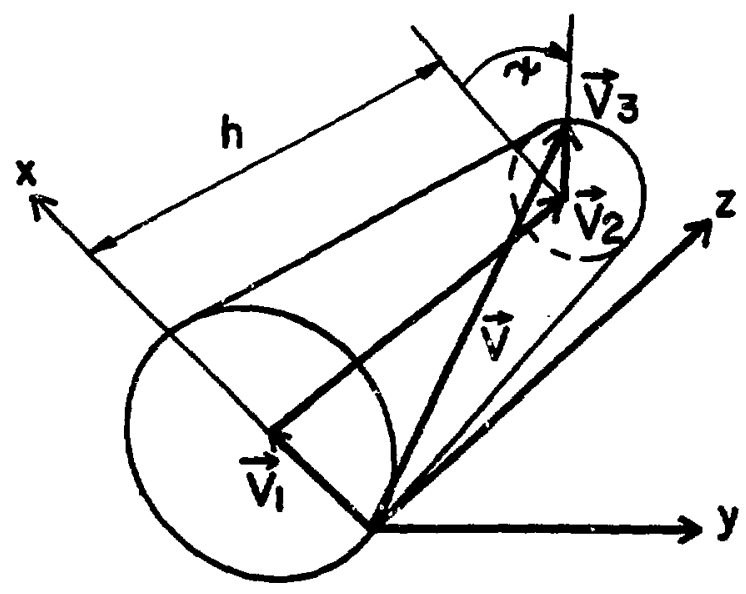

Fig. 6.

The vector $\vec{V}$ locating an arbitrary point on the small end of the frustum relative to the ryyz system. 
radii of the large and small ends of the frustum are given by $r_{1}$ and $r_{2}$, respectively. The angle $\psi$ is measured in the plane defined by the small end of the frustum and is measured from a line parallel to the $x$-axis to the vector $\vec{V}_{3}$. The frustum height is denoted by $h$. Thus, the vectors $\vec{v}_{1}, \vec{v}_{2}$, and $\vec{v}_{3}$ are as follows in the xyz system:

$$
\vec{v}_{1}=\left[\begin{array}{l}
r_{1} \\
0 \\
0
\end{array}\right]: \quad \vec{v}_{2}=\left[\begin{array}{l}
0 \\
0 \\
h
\end{array}\right]: \vec{v}_{3}=\left[\begin{array}{cc}
r_{2} & \cos \psi \\
r_{2} & \sin \psi \\
0
\end{array}\right] .
$$

Because

$$
\vec{v}=\vec{v}_{1}+\vec{v}_{2}+\vec{v}_{3}
$$

it follows that

$$
[\vec{v}]_{x y z}=\left[\begin{array}{c}
r_{1}+r_{2} \cos \psi \\
r_{2} \sin \psi \\
h
\end{array}\right]
$$

To find the vector $\vec{R}$ we must transform the coordinates of the vector $\vec{V}$ into the XYZ system. The orientation of the $x y z$ system relative to the $X Y Z$ system is defined by the Euler angles $\theta, \beta$, and $\gamma$. This then defines an orthogonal transformation matrix $T$ that transforms the coordinates of the vector $\vec{V}$ from the xyz system to the XYZ system, i.e.,

$$
[\vec{v}]_{X Y Z}=T[\vec{v}]_{X Y Z}
$$

where

$$
T=\left[\begin{array}{lll}
p_{1} & p_{4} & p_{7} \\
p_{2} & p_{5} & p_{8} \\
p_{3} & p_{6} & p_{9}
\end{array}\right]
$$

and

$$
\begin{aligned}
& \mathbf{p}_{1}=\cos \theta \cos \gamma-\sin \theta \sin \beta \sin \gamma, \\
& \mathbf{p}_{2}=-\sin \theta \cos \gamma-\sin \beta \sin \gamma \cos \theta, \\
& \mathbf{p}_{3}=-\sin \gamma \cos \beta, \\
& \mathbf{p}_{4}=\cos \beta \sin \theta, \\
& \mathbf{p}_{5}=\cos \theta \cos \beta, \\
& \mathbf{p}_{6}=-\sin \beta, \\
& \mathbf{p}_{7}=\cos \theta \sin \gamma+\sin \theta \sin \beta \cos \gamma, \\
& \mathbf{p}_{8}=-\sin \theta \sin \gamma+\cos \theta \sin \beta \cos \gamma,
\end{aligned}
$$

and

$$
\mathrm{P}_{9}=\cos \gamma \cos \beta \text {. }
$$

Substituting Eqs. (6), (7), (9), and (10) into Eq. (5) gives for the vector $\vec{R}$ in $X Y Z$ coordinates

$$
[\mathrm{k}]_{\mathrm{XYZ}}=r_{0}\left[\begin{array}{c}
\cos \theta \\
\sin \theta \\
0
\end{array}\right]+T\left[\begin{array}{c}
r_{1}+r_{2} \cos \psi \\
r_{2} \sin \psi \\
h
\end{array}\right] \text {. }
$$

The X-and Y-components of the vector $\vec{R}$ can then be used in Eq. (4) to solve for $r_{c}$.

The point on the small end of the frustum that yields $r_{c \max }$ is found by a one-dimensional search on the parameter $\psi$. Iteration on the Euler angle $\gamma$ is complete when $r_{c m a x}$ is equal to the outer beam radius. This is equivalent to the small end of the frustum being tangent to the circumference of the outer beam.

In step 4 of the iteration, angle $\beta$ is set to yield the desired angle $\phi$ between the pipe and the frustum axes. Now the frustum axis is parallel to the $z$-axis. Thus the cosine of the angle between the pipe and the frustum axes will be equal to the dot product of the unit vectors directed along the $Z$ - and $z$-axes. This is equivalent to element $p_{9}$ of the matrix $T$. Hence,

$$
\phi=\cos ^{-1}(\cos Y \cos B) \text {. }
$$

Linear iteration is performed on the angle $\beta$ until the actual inclination angle $\phi$ is equal to within $1 \sigma^{-6} \mathrm{rad}$ of the desired inclination angle.

With $\gamma$ and $\beta$ determined, the angle $\theta$ is computed by a linear iteration so that the surface of the frustum is tangent to the inner beam. This tangency point is determined in a manner similar to that used for determining the tangency point at the outer beam and small end of the 
frustum. Here, however, the tangency point can lie at any point on the frustum surface. Let $\vec{R}$ be the vector directed from the origin of the XYZ system to any point on the frustum surface. The geometry of Fig. 5 is applicable if the vector $\vec{R}$ is not restricted to the small end of the frustum. Let $\overrightarrow{\mathrm{U}}$ be the vector joining the $\mathrm{XYZ}$ and $\mathrm{xyz}$ systems, and let $\vec{v}$ be the vector from the origin of the $x y z$ system to the end point of the vector $\vec{R}$. Equations (5)-(7) are applicable here. The vector $\vec{V}$ can be expressed in terms of $x y z$ coordinates by a consideration of the frustum shown in Fig. 7. Again $\vec{V}$ is equal to the sum of the vectors $\vec{v}_{1}, \vec{v}_{2}$, and $\vec{v}_{3}$. The vector $\vec{v}_{1}$ is as defined in Eq. (8). The vector $\vec{v}_{2}$ is still directed along the $z$-axis but it is now of variable length $z_{p}$, i.e.,

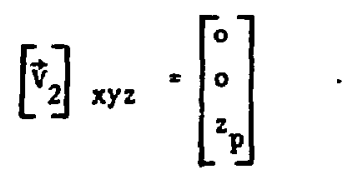

The vector $\vec{v}_{3}$ lies in a plane parallel to the $x y$ plane and is directed from the frustum centerline to the frustum surface. Let $r_{f}$ be the radius of the frustum at a cross section lying at a distance $z_{p}$ from the frustum base. Then

$$
r_{f}=r_{1}-\frac{z_{p}\left(r_{1}-r_{2}\right)}{h} \text {, }
$$

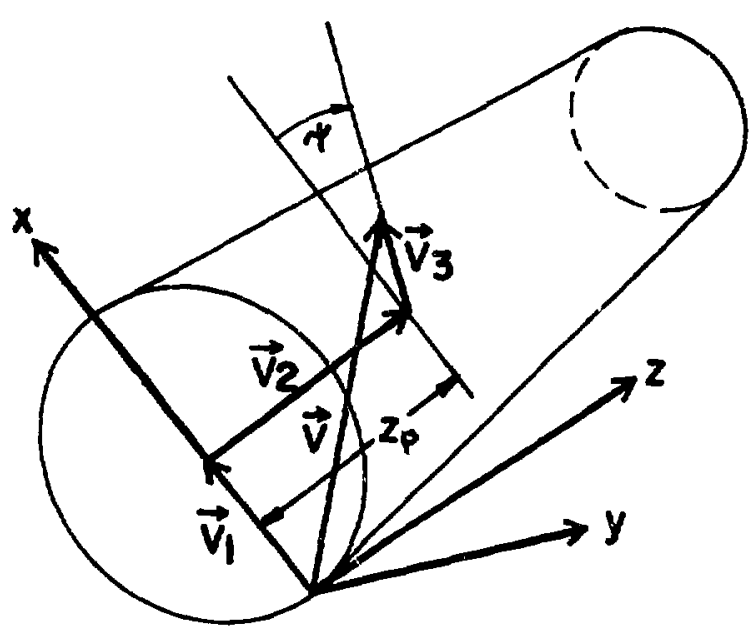

Fig. 7.

The vector $\vec{V}$ locating an arbitrary point on the frustum surface relative to the $x y z$ system. where

$r_{1}=$ the radius of the frustum base (large end),

$r_{2}=$ the radius of the small end of the frustum,

and

$h=$ the height of the frustum.

With $\psi$ defined as the angle in a plane parallel to the $x y$ plane and measured between a line parallel to the $x$-axis and the vector $\vec{v}_{3}$, it follows that

$$
\left[\vec{v}_{3}\right]_{x y z}=\left[\begin{array}{c}
x_{f} \cos \psi \\
r_{f} \sin \psi \\
0
\end{array}\right] \text {. }
$$

Summing the vectors $\vec{v}_{1}$ from Eq. (8), $\vec{v}_{2}$ from Eq. (13), and $\vec{v}_{3}$ from Eqs. (14) and (15) gives for the vector $\vec{v}$

$$
[\vec{v}]_{x y z}=\left[\begin{array}{c}
r_{1}+\left[r_{1}-\frac{z_{p}\left(r_{1}-r_{2}\right)}{h}\right] \cos \psi \\
\left.r_{1}-z_{p} \frac{\left(r_{1}-r_{2}\right)}{h}\right] \sin \psi \\
z_{p}
\end{array}\right] .
$$

The vector $\vec{R}$ can now be obtained by transforming the vector $\vec{V}$ by way of the matrix $T$ and adding the result to the vector $\vec{U}$ defined by Eqs. (5) and (6), i.e.,

[]$_{X Y Z}=r_{0}\left[\begin{array}{c}-\cos \theta \\ \sin \theta \\ 0\end{array}\right]+\left[\begin{array}{c}r_{1}+\left[r_{1}-z_{p} \frac{\left(r_{1}-r_{2}\right)}{h}\right] \cos \psi \\ {\left[r_{1}-z_{p} \frac{\left(r_{1}-r_{2}\right)}{h}\right] \sin \psi} \\ z_{p}\end{array}\right]$. 
As seen from Eq. (17), for fixed angles $\theta, \beta$, and $\gamma$, the vector $\vec{R}$ is $\approx$ function of the distance $z_{p}$ and the angle $\psi$. We now find that point on the frustum surface that lies closest to the pipe centerline. With

$r_{c f}=$ to the perpendicular distance from the pipe centerline to the frustum surface

it follows that

$$
\mathbf{r}_{\text {cf }}=\left(R_{X}{ }^{2}+R_{Y}{ }^{2}\right)^{1 / 2} .
$$

The minimum value of $r_{c f}, r_{c f m i n}$, is the minimum distance between the pipe centerline and the frustum surface. Thus, if $r_{\text {cfmin }}$ is less than the inner beam radius, a portion of the frustum surface lies inside the inner beam, whereas if $\mathbf{r}_{\mathbf{c f m}}$ in is greater than the inner beam radius, there is no common point between the frustum and the inner beam. When $\mathbf{r}_{\mathrm{cfmin}}$ is equal to the inner beam radius, the inner beam and the 5 rum surface are tangent. Therefore, we must compute $r_{\text {cfmin }}$.

For fixed angles $\theta, \beta$, and $\gamma$ the distances $R_{x}$ and $R_{y}$ are functions of $z_{p}$ and $\psi$. Hence, $r_{c f m i n}$ can be found by minimizing $r_{c f}$ with respect to the parameters $z_{p}$ and $\psi$ subject to the constraint that the minimum point actually lies upon the frustum. Let $\mathrm{J}$ be the function of $\mathrm{z}_{\mathrm{p}}$ and $\psi$ to be minimized. Then

$$
\begin{aligned}
J & =\left(R_{x}{ }^{2}+R_{y}{ }^{2}\right)^{1 / 2} \\
& +K\left[s\left(z_{p}-h\right)\left(z_{p}-h\right)^{2}+s\left({ }_{p}\right) z_{p}^{2}\right],
\end{aligned}
$$

where

$$
K=\text { large penalty constant }
$$

and

$$
s(f)=\text { step function }=\left\{\begin{array}{l}
1 \mathrm{f}>0 \\
0 \mathrm{f}<0
\end{array}\right\} .
$$

Note from Eq. (19) that the function $J$ is composed of the quantity to be minimized $\left(r_{c f}=\sqrt{R_{x}^{2}+R_{y}^{2}}\right)$ and a penalty term to ensure that the minimum does not lie at some value of $z_{p}$, which is not on the frustum surface. The values of $z_{\mathrm{p}}$ and $\psi$, which minimize the function $\mathrm{J}$ and consequently $r_{c f}$, are obtained with the aid of a Davidon iterator. The Davidon iterator is a second-order method for finding the minimum of a multiparameter function. Rapid and reliable convergence to the minimum can be obtained with this iterator for the type function J defined by Eq. (19). With the minimum of $r_{c f}$ coniputed in this way, linear iteration on the angle $\theta$ is executed until the difference between $r_{c f m i n}$ and the inner beam radius is less than $10^{-6}$ in.

\section{COMPUTATION OF THE FRUSTUM SUPPORT GEOMETRY}

Exact determination of the frustum location and orientation has been outlined above. As shown in Fig. 2, the frustum is mounted on a flat plate that, in turn, is supported by four support legs welded to the pipe wall. The location and orientation of the frustum relative to the support plate and the location of the legs relative to the support plate are prespecified quantities. We must determine the dimensions of the support legs and the points at which the support legs should be welded to the pipe.

A typical support leg is shown in Fig. 8. The top of the support leg is fastened perpendicular to the support plate while the bottom surface of the support leg rests on the inside surface of the test pipe. All cross-sectional planes of the support leg that are parallel to the top are squares of width $w$. We must find the lengths $\ell_{1}, \ell_{2}, \ell_{3}$, and $\ell_{4}$ of the support leg edges so that the support legs will rest firmly on the pipe surface and at the same time provide the proper location and orientation of the frustum. In addition, the points at which these edges intersect the

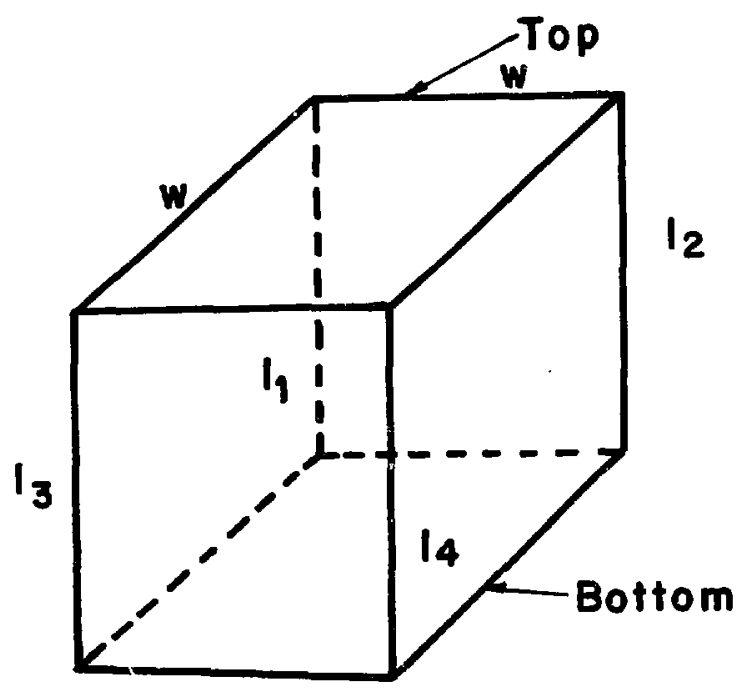

Fig. 8.

Support leg geometry. 
pipe are needed to locate the support plate properly within the pipe.

A support leg edge, along with the support plate and pipe, is shown in Fig. 9. The XYZ-coordinate system,with origin at the pipe center and the $Z$-axis directed along the pipe centerline, is identical to the $X Y Z$ system previously defined. The $x^{\prime} y^{\prime} z^{\prime}$-coordinate system shown in Fig. 9 is fixed to the support plate with the origin of the system lying in the center of the plate bottom. The $x^{\prime}$-axis is normal to the plate surface and the $z^{\prime}$-axis is directed along the centerline of the bottom surface. The origins of the $X Y Z$ and $x^{\prime} y^{\prime} z^{\prime}$ systems lie in the same cross-sectional plane of the pipe. The $x^{\prime} y^{\prime} z^{\prime}$ system can be derived from the $\mathrm{xyz}$ system by translating the origin of the $\mathrm{xyz}$ system from the bottom of the frustum to the bottom of the support plate and by rotating this translated system about the $y$-axis through some angle $\eta$ so that the $z^{\prime}$-axis lies in the bottom surface of the plate. Thus, the Euler angles that define the orientation of the $x^{\prime} y^{\prime} z$ ' system relative to

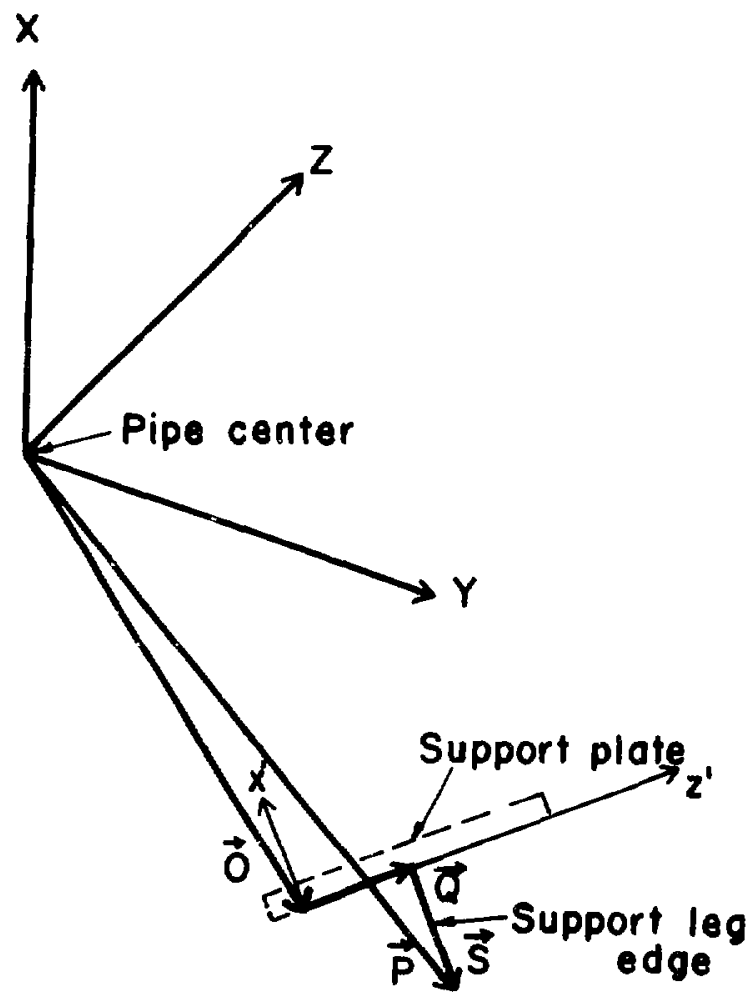

Vectors $\vec{O}, \vec{P}, \vec{Q}$, and $\vec{S}$ defining the extremities of $a$ support leg edge relative to the support plate and the pipe center. the $\mathrm{XYZ}$ system are $\theta, \beta$, and $\gamma^{\prime}$, where $\theta$ and $\beta$ are the same as defined in Sec. III and

$$
r^{\prime}=r+n
$$

Let $\mathbf{T}^{\prime}$ be the matrix that transforms the coordinates of a vector from the $x^{\prime} y^{\prime} z^{\prime}$ system to the $X Y Z$ system. Then $T^{\prime}$ can be obtained from the matrix $T$ defined in Sec. III by replacing $\boldsymbol{\gamma}$ with $\boldsymbol{\gamma}^{\prime}$.

The vector $\vec{O}$ shown in lig. 9 locates the origin of the $x^{\prime} y^{\prime} z$ ' system relative to the $X Y Z$ system, and the sum of the vectors $\vec{Q}$ and $\vec{S}$ locates the extremity of the support leg edge relative to the origin of the $x^{\prime} y^{\prime} z$ system. The vcctor $\overrightarrow{\mathbf{P}}$ locates the extremity of the edge relative to the $\mathrm{XYZ}$ system. Therefore, the point at which the edge intersects the pipe is defined by the coordinates of $\overrightarrow{\mathbf{P}}$, and the length of the edge is equal to the length of $\vec{S}$.

We must determine the length of the vector $\vec{S}$ so that the end point of $\vec{S}$ touches the inner wall of the pipe. This is equivalent to requiring that the radial distance of the end point of $\overrightarrow{\mathbf{S}}$ from the center of the pipe be equal to the pipe radius. Let $P_{\mathbf{X}}$ and $P_{\mathbf{Y}}$ be the $X$ - and $\mathbf{Y}$-coordinates, respectively, of $\overrightarrow{\mathrm{P}}$. Then the radial distance from the center of the pipe will equal $\left(P_{X}^{2}+P_{Y}^{2}\right)^{1 / 2}$. Hence, we must determine the length of the vector $\vec{S}$ so that

$$
\text { pipe radius } \cdot\left(\mathrm{P}_{X}^{2}+\mathrm{P}_{Y}{ }^{2}\right)^{A} \text {. }
$$

From Fig. 9 we know that $\overrightarrow{\mathbf{P}}$ is the sum of the vectors $\overrightarrow{\mathbf{O}}, \overrightarrow{\mathbf{Q}}$, and $\overrightarrow{\mathbf{S}}$, i.e.,

$$
\Phi=\boldsymbol{t}+\boldsymbol{\nabla}+\mathbf{s} \text {. }
$$

The vector $\overrightarrow{0}$ can be represented as the sum of the vectors $\overrightarrow{\mathrm{U}}$ and $\overrightarrow{\mathrm{G}}$ (see Fig. 10), where the vector $\overrightarrow{\mathrm{U}}$ is directed from the origin of $X Y Z$ to the origin of $x y z$, and the vector $\vec{G}$ is directed from the origin of $x y z$ to the origin of $x^{\prime} y^{\prime} z$. Both vectors $\vec{U}$ and $\vec{G}$ lie in the $X Y$ plane with the $X$ - and $Y$-coordinates of $\vec{U}$ given by Eqs. (6) and (7). The vector $\vec{G}$ has a specified length equal to $g$ and is directed along the negative $x^{\prime}$-axis, i.e.,

$$
[\vec{G}]_{x^{\prime} y^{\prime} z^{\prime}}=\left[\begin{array}{c}
-g \\
0 \\
0
\end{array}\right] \text {. }
$$




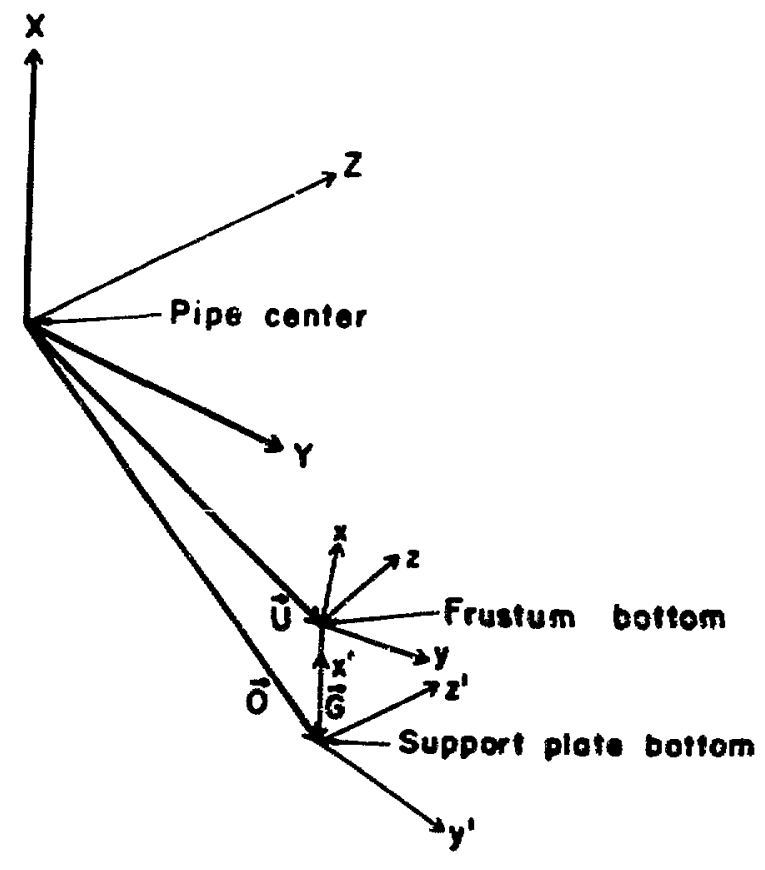

Fig. 10.

Representation of the vector $\vec{O}$ as the vector sum of $\vec{U}$ and $\vec{G}$.

Transforming the vector $\overrightarrow{\mathbf{G}}$ into the $X Y Z$ system by means of the matrix $T^{\prime}$ and forming the vector $\vec{O}$ as the vector sum of $\overrightarrow{\mathbb{U}}$ and $\overrightarrow{\mathbf{G}}$ gives

$$
[\delta]_{X Y Z}=\left[\begin{array}{cc}
-x_{0} & \cos \theta \\
x_{0} & \sin \theta \\
0
\end{array}\right]+T \cdot\left[\begin{array}{c}
-\theta \\
0 \\
0
\end{array}\right] \text {. }
$$

The vector $\vec{\Omega}$ shown in Fig. 9 provides the location of the support edge relative to the plate. Hence, the $y^{\prime}$ - and $z^{\prime}$-coordinates of $\mathbf{Q} Q_{y}$, and $\mathbf{Q}_{2}$ are readily obrainable as input to the alignment problem. Obviously the $x^{\prime}$-component of $\vec{Q}$ will be zero because $\vec{Q}$ lies in the bottom of the support plane and $x^{\prime}$ is normal to this plane, i.e.,

$$
[Q] x^{\prime} y^{\prime} z^{\prime} \cdot\left[\begin{array}{c}
0 \\
g_{1} \\
q_{z},
\end{array}\right] \text {. }
$$

where $Q^{\prime}$ and $g_{2}$ are known values.

The vector $\vec{s}$ has a component only in the negative $x^{*}$ direction because this vector is normal to the suppoz plate and therefore nomal to lif $y^{\prime} z$ " plane. Hente, s sth be represented in the $x^{\prime} y^{\prime \prime} z^{6}$ system as follows:

$$
[3]_{x+y+z} \cdot\left[\begin{array}{c}
-1 \\
0 \\
0
\end{array}\right] \text {. }
$$

where $L$ equals the unkrigwn tength of the treeror $\vec{S}$.

Transforming $\vec{Q}$ and $\vec{S}$ from Eqs. (25) and (26), nspec. tively, by means of the matrix and substituring thes transformed vectors along with the sector $\overrightarrow{0}$ from $E q$. (24) into Eq. (22) gives for the vecto: $\vec{p}$

$$
[F]_{X Y Z}=\left[\begin{array}{cc}
-\tau_{0} & \cos \\
\tau_{0} & \sin \\
0
\end{array}\right] \cdot \tau^{\prime}\left[\begin{array}{c}
-t-t \\
\theta_{\gamma} \\
\theta_{2}
\end{array}\right]
$$

With the expression for $\overrightarrow{\mathbf{P}}$ as above, a linear iteration on the lengrh $L$ is carried out until Eq. (21) is satisfied to within $1 \sigma^{6}$ in. This yields the desired kength of the support $\mathrm{lg}$ edige. Also, the coordinates of the vector $\bar{P}$ define the point where the support leg edge touches the inside of the rest pipe. 


\section{v. Conclustons}

This incation acthrique has worked sutecesfully in whing the probien of aliphing the loustum of acone in

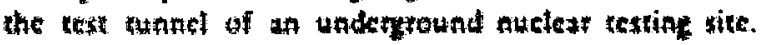

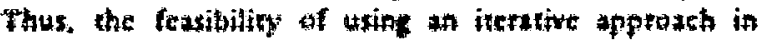
conjunetion with a bightoed digitat computet to hathlk

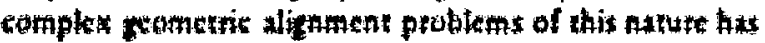
been proted. Antid contergence of the iterator was experianed with the conl computation time on the coc 6600 eomputef beiny kss than 1 sec.

Sirte precision arithenetic was ded throughour the compuration vo conserve computer ture starke on the

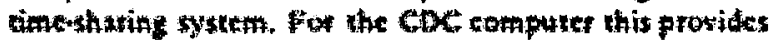

about if decimal ditits of atctatary. The Davidon iterator hat to be "funed" to operate within these aceutzey

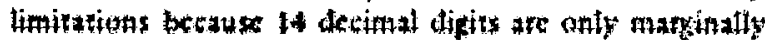

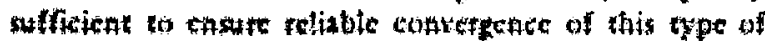

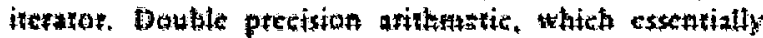
dowbles the numbet of accurate decimal digits, would have ctiminated these difficuthics.

\section{ACKNOWLEDGWENTS}

1 wish to than fohn Quitein of THW systems, thaston. for polyine a rey well texted computer pro.

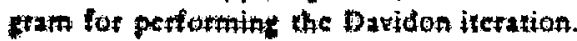

